\title{
Transient and Permanent Rotations in a Shear Layer Excited by Strong Earthquake Pulses
}

\author{
by V. Gi ev and M. D. Trifunac
}

\begin{abstract}
Nonlinear waves in a bilinear soil layer are described for excitation by vertically arriving $\mathrm{S}$-wave pulses of strong ground motion. Conditions that lead to the nonlinear deformation are described in terms of amplitudes and wavelengths of incident pulses. It is shown that the layer can fail during the first passage of the incident wave (during a time shorter than the travel time through the layer). Peak amplitudes of (1) transient rotations, of (2) permanent rotations (strains), and of (3) the peak ductility in the layer are described in terms of the dimensionless amplitudes of incident pulses and the places of their occurrence in the layer. Even a simple model like this (one-dimensional propagation, simple shape of incident pulse, bilinear stress-strain soil model) leads to very complicated response. The results presented offer only a glimpse at the complexity in a realistic setting.
\end{abstract}

\section{Introduction}

Before the era of modern seismology (early 1900s) and well before the first observations of strong ground motion in 1932 (Trifunac, 2008), it was known that nonlinear response of sediments can lead to profound changes in the earthquake ground motion relative to the basement rock (Milne, 1898). The evidence that soils have complex nonlinear properties also came from the laboratory experiments (Terzaghi, 1925) involving both static and dynamic loads, but it would take several more decades before earthquake engineers started to investigate dynamic soil properties (e.g., Seed and Idriss, 1970; Hardin and Drnevich, 1972) and nonlinear soil response to earthquake shaking. In contrast, the first part of twentieth century seismological research, which focused on the studies of the earth interior and teleseismic observation and data analysis, led to the prevailing view that most observations of earthquake motion can be described in terms of the linear material properties and elastic waves. It was not until after the occurrence of the Northridge 1994, Kobe 1995, and Nisqually 2001 earthquakes, which contributed the nearfield measurements on widespread nonlinear sediment response, that nonlinear site response started to attract the attention of many seismologists as well. The papers by Safak (2001) and Hartzell et al. (2004) review the models, the nonlinear hysteretic representation of soil, and the methods of data analysis and their interpretation in many of those recent studies.

With the exception of soft (low-velocity) and cohesionless soils, most sites at moderate and large distances respond to shaking by intermediate and small earthquakes in an essentially linear manner, but above the strains of $10^{5}$, the nonlinear response in typical soils is initiated (Trifunac et al., 1996, 1999; Trifunac and Ivanovi , 2003a,b). Large non- linear responses of soils near the ground surface (liquefaction, landslides) are detected by surveys following an earthquake, but nonlinear responses at depth are more difficult to identify. The evidence of nonlinear response of soil deposits can be seen in the reduction of the measured peak amplitudes of strong ground motion (Trifunac and Todorovska, 1996) in the reduction of damage to buildings, in the distribution of damaged and broken water and gas pipes (Trifunac and Todorovska, 1998), and in the surface manifestations in the epicentral area (Earthquake Engineering Research Institute, 1995).

Observations of the nonlinear phenomena in soilstructure interaction show that the nonlinearity first begins to occur in the soil surrounding the building foundation (Trifunac et al., 2001a,b). Complex nonlinearities in the large blocks of soil and in the soil-structure systems also occur when the gaps are opened in the soil or along the soilstructure contact surfaces during large shaking amplitudes. These gaps can remain open for extended periods of time and when shaken by subsequent earthquakes, they can be reactivated and changed (Trifunac and Todorovska, 2004). The absence of recorded rotational components of strong motion makes it impossible to infer the permanent displacements and permanent tilting of the building foundations and of the soil blocks in the heavily shaken areas after earthquakes. These complexities and the strong dependence of the outcome on the time-dependent nature of each strong earthquake shaking make the attempts to further develop seismic zoning maps that consider nonlinear site response a difficult challenge (Trifunac and Todorovska, 2000a,b).

Wave-propagation methods in earthquake engineering analyses of the linear response of soil layers and of buildings 
have been used since the 1930s (Sezawa and Kanai, 1935, 1936; Kanai, 1965, 1983). In the following, the elementary aspects of wave propagation through a homogeneous shear layer will be used to study the relationships among the amplitudes of incident pulses and of the layer response with emphasis on transient and permanent rotations. Onedimensional (1D) representation of nonlinear shear waves will be used. Because this model also describes shear waves in long buildings (when rocking response associated with soil-structure interaction can be neglected), some of the results of this analysis may also be useful for understanding the elementary aspects of nonlinear response of such buildings (Gi ev and Trifunac, 2007a,b).

The rotational components of earthquake ground motion measured by Takeo (2006) several kilometers from the faults of moderate earthquakes were found to be larger than what would be predicted by numerical simulations using the classical linear theory. Takeo and Ito (1997) have shown that this discrepancy could be reduced by considering additional terms in the representation of the earthquake source, but observations of widespread surface deformations in the heavily shaken epicentral regions of shallow earthquakes indicate that nonlinear response of surface soil must also be considered in the analysis of the observed and recorded strong motion (Trifunac, 2009). To explore some elementary aspects of this problem, we present in the following an analysis of nonlinear shear waves in a surface layer, and we focus our attention on transient and permanent rotations caused by those waves.

We will illustrate only some elementary characteristics of 1D nonlinear waves in a single layer with bilinear stiffness properties, overlying an elastic half-space and excited by S-wave pulses arriving vertically up through the half-space. We assume the bilinear constitutive law because of its simplicity and because it resembles the variety of constitutive laws proposed by different authors (e.g., Joyner and Chen, 1975; Pyke, 1979; Finn, 1982; Iai et al., 1990; Vu eti , 1990; Li and Liao, 1993; Bonilla, 2000). Our hysteresis loops follow the Massing criteria (Massing, 1926; Kramer, 1996) and are defined by three parameters: initial slope ${ }_{1}$, the yielding strain in the soil ${ }_{\mathrm{yb}}$, and the second slope $1 \quad 0 \geq 0$. Our aim is to describe how the amplitudes and duration of a simple incident pulse lead to creation of large strains (rotations), permanent deformation, and permanent tilting and to understand the conditions that govern their location inside the layer. Because most engineering structures have foundations that are embedded several to several tens of meters, an understanding of the permanent deformations and tilts in a simple, relatively soft layer of soil should provide a useful starting point for understanding some of the observed damage in the near field. It should also help in the development of soilstructure models with nonlinear soil properties. Because of the complexities of the large nonlinear response, we limit the scope of this study to the response of one layer only. Throughout this article we will use rotations and strains in- terchangeably, and we will consider only small deformations for which $\tan$.

\section{Model and Numerical Scheme}

We study horizontal shear deformations, $u$, in a 1D soil layer supported by a half-space and excited by a vertically propagating shear wave represented by a half-sinepulse (Fig. 1). A finite difference scheme for solution of this problem with accuracy $0 \quad t^{2} ; \quad x^{2}$, where $x$ and $t$ are the space and time increments, leads to the exact solution when $t=x \quad 1$, where is the velocity of shear waves (Gi ev and Trifunac 2007b). With a ratio of the spatial intervals $x_{b}=x_{s} \quad b=s$, this requirement can be satisfied, where $\mathrm{X}$ is the coordinate along the wave propagation. The subscripts $\mathrm{b}$ and $\mathrm{S}$ designate the values in the layer with thickness $\mathrm{H}_{b}$ and in the half-space, respectively. The equation of motion is

$$
\mathrm{v}_{\mathrm{t}} \quad \mathrm{x}=\text {; }
$$

and the relation between the derivative of the strain and the velocity is

$$
\text { t } \quad v_{x} ;
$$

where $\mathrm{V}$, , and are particle velocity, density, shear stress, and shear strain, and the subscripts $t$ and $x$ represent derivatives with respect to time and space.

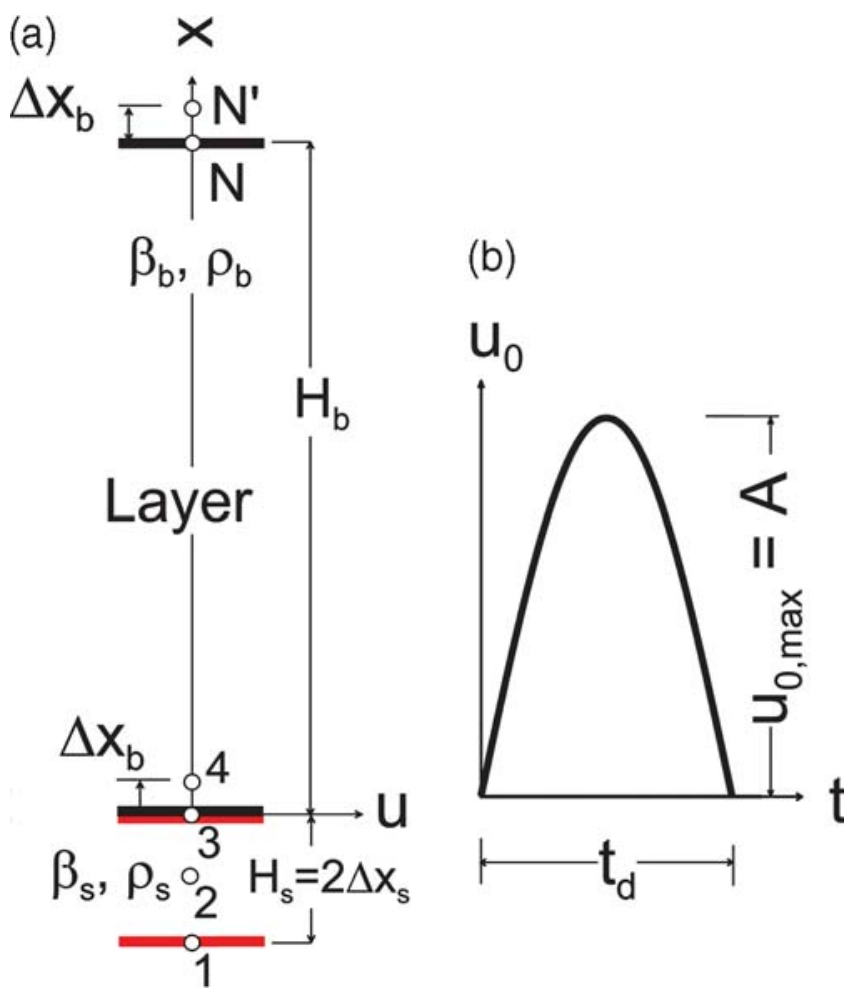

Figure 1. Soil layer and incoming strong-motion displacement pulse: (a) model of the soil layer and (b) the pulse in the half-space. 
The domain for analysis consists of two materials (see Fig. 1): $2 \quad x_{s} \leq x<0$ with physical properties $s$ and $s$ representing the half-space and $0<x \leq H_{b}$ with physical properties $b$ and $b$ representing the soil layer. $v \quad \partial u=\partial t$ and $\quad \partial u=\partial x$ are the velocity and the strain at a point, and $\mathrm{u}$ is out-of-plane displacement of a particle perpendicular to the propagation ray. It is assumed that the incoming wave is known and that its displacement as a function of $t$ is prescribed at point $1\left(\begin{array}{lll}x & 2 & x_{s}\end{array}\right)$. It is also assumed that the half-space is always in the linear elastic state. The LaxWendroff (1964) finite difference method for a set of simultaneous equations is used to solve the problem. A mesh with different spatial intervals in the half-space and in the soil layer is used. The spatial intervals are defined by $x_{i}$ $i$. $t$, where $i$ is the velocity of shear waves in the half-space ( $\left.\begin{array}{lll}i & s\end{array}\right)$ or in the soil layer ( $\left.i \quad b\right)$, and $t$ is the timestep. The soil layer is discretized into 197 spatial intervals and the half-space into two, so the properties of the mesh are

$$
x_{b} \quad H_{b}=197 ; \text { and } x_{s} \quad x_{b} s_{b} \text { : }
$$

The cell length at the interface point $3\left(\begin{array}{ll}x & 0\end{array}\right)$ is

$$
x_{3} \quad x_{b} \quad x_{s}=2 \text {; }
$$

and the initial equivalent shear modulus for this point is obtained from the condition of continuity of the displacements and the stresses at the interface as

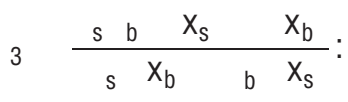

The yielding strain in the soil layer is $y b$, and to maintain the continuity of the stresses at the interface, the yielding strain in contact point 3 is obtained from $\quad$ b yb 3 yз (Fig. 2)

$$
\text { y3 } \quad \text { yb } \quad \text { b }=3 \text {; }
$$

where $\quad b$ is the shear modulus in the layer, and ${ }_{3}$ is the equivalent shear modulus at contact point 3 . The timestep is constant during the analysis,

$$
\text { t } \quad x_{b}=b_{b} \quad x_{s}=s:
$$

Above the top point $\mathrm{N}$ (free surface of the layer), an additional point $N^{\prime}$ is introduced at a distance of $x_{b}$. For a stress-free point $\mathrm{N}$ for all time, the velocities and the stress at $\mathrm{N}^{0}$ are updated as

$$
\begin{array}{llll}
\mathrm{V}_{\mathrm{N}^{0}} & \mathrm{~V}_{\mathrm{N}} & 1 \\
\mathrm{~N}^{1} & \mathrm{~N}_{1} & 1:
\end{array}
$$

Equation (1) can be written in vector form as

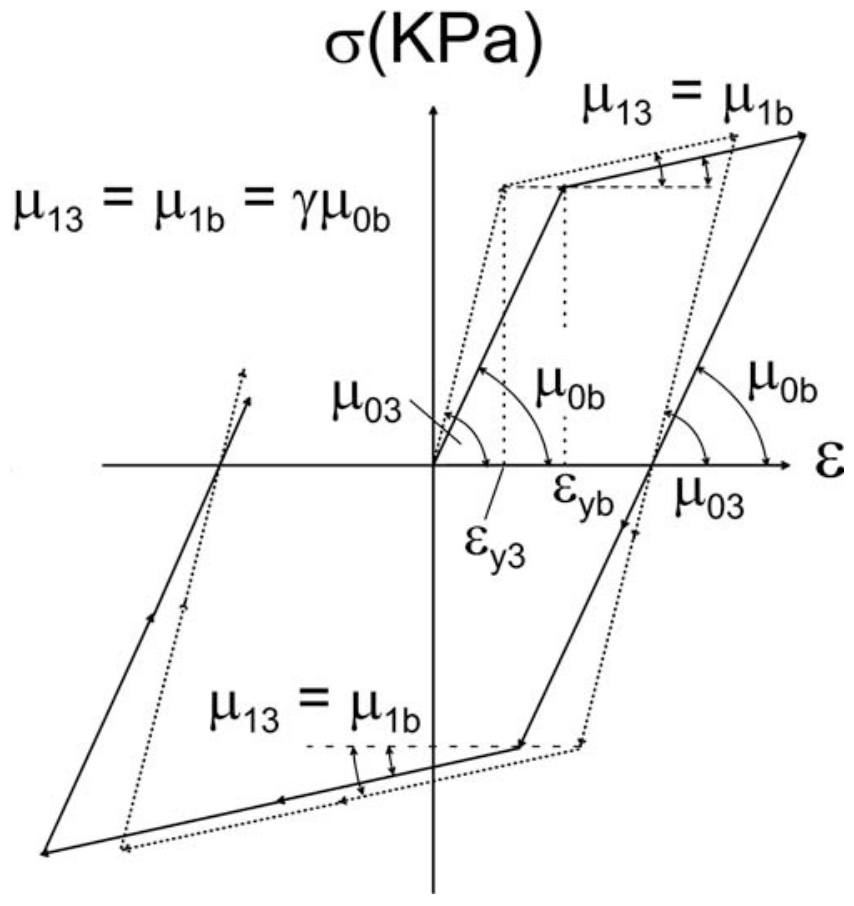

Figure 2. The constitutive laws, , for the soil layer (solid line) and for the interface (dotted line).

$$
\frac{\partial \mathbf{U}}{\partial t} \quad \frac{\partial \mathbf{F}}{\partial x}
$$

where

$$
\mathbf{U}\left\{\begin{array}{l}
\mathrm{v} \\
\}
\end{array} \text { and } \mathbf{F}\left\{\begin{array}{c}
- \\
\mathrm{v}
\end{array}\right\} \quad\left\{\frac{-}{\mathrm{v}}\right\}\right. \text { : }
$$

The vector $\mathbf{U}$ at point $\mathrm{i}$ in time $\mathrm{j} \quad 1 \quad \mathrm{t}$ expanded in Taylor series is

$$
\mathbf{U}_{i ; j \quad 1} \quad \mathbf{U}_{i ; j} \quad t\left(\frac{\partial \mathbf{U}}{\partial t}\right)_{i ; j} \quad \frac{t^{2}}{2}\left(\frac{\partial^{2} \mathbf{U}}{\partial t^{2}}\right)_{i ; j} \quad 0 \quad t^{3}
$$

and from equation (7)

$$
\begin{aligned}
& \mathbf{U}_{i ; j} 1 \quad \mathbf{U}_{i ; j} \quad t\left(\frac{\partial \mathbf{F}}{\partial x}\right)_{i ; j} \quad \frac{t^{2}}{2} \frac{\partial}{\partial t}\left(\frac{\partial \mathbf{F}}{\partial x}\right)_{i ; j} \quad 0 \quad t^{3} \\
& \mathbf{U}_{i ; j} 1 \quad \mathbf{U}_{i ; j} \quad t\left(\frac{\partial \mathbf{F}}{\partial x}\right)_{i ; j} \quad \frac{t^{2}}{2} \frac{\partial}{\partial x}\left(\mathbf{A} \mathbf{U} \frac{\partial \mathbf{F}}{\partial x}\right)_{i ; j} \\
& 0 \quad t^{3} \text { : }
\end{aligned}
$$

A $\mathbf{U}$ is the Jacobian matrix

$$
\mathbf{A} \mathbf{U} \quad \frac{\partial \mathbf{F}}{\partial \mathbf{U}} \quad\left[\begin{array}{ll}
\frac{\partial}{\partial v} & \frac{\partial}{\partial} \\
\frac{\partial v}{\partial v} & \frac{\partial v}{\partial}
\end{array}\right] \quad\left[\begin{array}{cc}
0 & 1 \frac{d}{d} \\
1 & 0
\end{array}\right] \text { : }
$$




\section{Transparent Boundary}

The transparent boundary adopted for this study follows the formulation of Fujino and Hakuno (1978) and Gi ev and Trifunac (2007b). It is a perfect transparent boundary for 1D waves when $t=x \quad 1$. For the linear case at the contact (point 3), one part of the incoming wave is transmitted into the other medium, and one is reflected back into the same medium. The corresponding coefficients are obtained from the boundary conditions of continuity of the displacements and stresses at the contact. For a transmitted wave from medium $B$ to medium $A$ and for a reflected wave from medium $B$ back into medium $B$, these coefficients are

$$
\mathrm{k}_{\mathrm{tr} B \rightarrow \mathrm{A}} \quad 21 \quad \text { a } \quad \mathrm{a}=\begin{array}{llll} 
& \mathrm{b} & \mathrm{b} & 1
\end{array}
$$

and

$$
\mathrm{k}_{\mathrm{ref} B \rightarrow \mathrm{B}} \quad 1 \quad \mathrm{a} \quad \mathrm{a}=\mathrm{b} \quad \mathrm{b}=1 \quad \mathrm{a} \text { a }=\mathrm{b} \quad \mathrm{b}:
$$

For the opposite direction of propagation, the numerators and the denominators in these fractions exchange places. For a wave arriving from the half-space, $B$ represents half-space and $A$ the soil layer. For a wave in the soil layer propagating down, $B$ represents the layer, and $A$ the half-space.

\section{Numerical Examples}

For the numerical analysis, we consider a soil layer supported by an elastic half-space. The densities of the halfspace and of the layer are assumed to be the same: b

s $\quad 2000 \mathrm{~kg}=\mathrm{m}^{3}$. The velocity of the shear waves in the half-space is taken as s $250 \mathrm{~m}=\mathrm{sec}$ and in the layer as b $100 \mathrm{~m}=\mathrm{sec}$. This example will illustrate the nonlinear response of a soft soil layer (with $\mathrm{H}_{\mathrm{b}} \quad 10 \mathrm{~m}$ and yb 0:02) overlying a sedimentary deposit.

To describe nonlinear response and the development of permanent deformations in the soil layer, we introduce two dimensionless parameters,

$$
\text { dimensionless amplitude } \quad A=H_{b} \cdot{ }_{y b} \text {; }
$$

where $A$ is the amplitude of the pulse (Fig. 1), $H_{b}$ is the thickness of the layer, yb is the yielding strain in the layer, and

dimensionless frequency

$$
2 \mathrm{H}_{b}={ }_{b} \quad 2 \mathrm{H}_{b}=b \cdot 2 t_{d} \quad H_{b}=b \cdot t_{d} ;
$$

where $\quad b$ is the wavelength of the wave in the layer, $\quad b$ is the shear-wave velocity in the layer, and $t_{d}$ is the duration of the incident wave represented by a half-sine pulse (Fig. 1b).

To understand the initiation of the permanent strain in the layer, first we examine the solution for the linear case. The displacement and the strain (rotation) in the linear layer are

$$
\begin{aligned}
& \text { u x; t } \quad A \sum_{j=1}^{\infty} k_{j}\left\{\sin \frac{\pi}{t_{d}}\left(\begin{array}{llll}
t & t_{j} & 1 & \frac{x}{b}
\end{array}\right)\right. \\
& \left.\times\left[\begin{array}{lllll}
H & t & t_{j} & 1 & \frac{x}{b}
\end{array}\right) \quad H\left(\begin{array}{lllll}
t & t_{j} & 1 & \frac{x}{b} & t_{d}
\end{array}\right)\right] \\
& \sin \frac{\pi}{t_{d}}\left(\begin{array}{lll}
t & t_{j} & \frac{x}{b}
\end{array}\right)\left[H\left(\begin{array}{lll}
t & t_{j} & \frac{x}{b}
\end{array}\right)\right. \\
& \left.\left.H\left(\begin{array}{llll}
t & t_{j} & \frac{x}{b} & t_{d}
\end{array}\right)\right]\right\}
\end{aligned}
$$

and

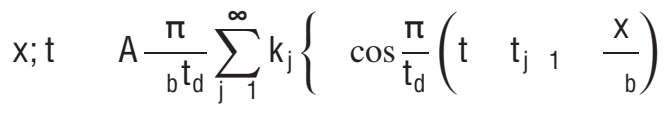

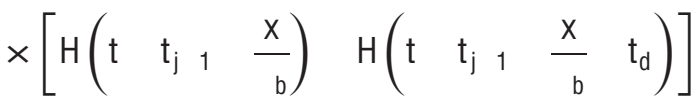

$$
\begin{aligned}
& \cos \frac{\pi}{t_{d}}\left(\begin{array}{lll}
t & t_{j} & \frac{x}{b}
\end{array}\right)\left[H\left(\begin{array}{lll}
t & t_{j} & \frac{x}{b}
\end{array}\right)\right. \\
& \left.\left.H\left(\begin{array}{llll}
t & t_{j} & \frac{x}{b} & t_{d}
\end{array}\right)\right]\right\} \text {; }
\end{aligned}
$$

where $j$ is the order number of the passage of the wave on the path bottom-top-bottom in the layer, $t_{j} \quad 2 \mathrm{jH}_{b}={ }_{b} j$ $0 ; 1 ; 2 ; 3 ; \ldots ;$ is the time required for the wave to pass $j$ times the path bottom-top-bottom (two heights), and

$$
k_{j} \quad k_{t} k_{r}^{j} 1
$$

is the amplitude factor of the pulse in the half-space during its $j$ th passage along the path bottom-top-bottom through the layer with $\mathrm{k}_{\mathrm{t}}$ and $\mathrm{k}_{\mathrm{r}}$ defined by equations (10) and (11).

The odd terms in the series of equations (14) and (15) describe the response to the pulse coming from below, while the even terms describe the response for the pulse arriving from above. For the shear-wave velocities in our example ( s $250 \mathrm{~m}=\mathrm{sec}$ and b $100 \mathrm{~m}=\mathrm{sec}$ ), $\mathrm{k}_{\mathrm{t}} \quad 10=7$, and $k_{r} 3=7$. In equation (14) the displacement is positive for odd passages and negative for even passages. The displacement and velocity change sign after reflection from the layer-half-space interface and do not change sign after reflection from the top of the layer. The strain changes sign after reflection from the top of the layer and does not change sign after reflection from the half-space (Fig. 3a). Figure 3b,c illustrates the changes in the nonlinear response when $\quad 0: 1$ and 0.3. The constant that multiplies the series in equation (15) in terms of dimensionless amplitude and dimensionless frequency is

$$
A \pi={ }_{b} t_{d} \quad A \quad \pi \quad y b:
$$

To analyze the occurrence of permanent strain, we consider two characteristic points in the layer: point $\mathrm{B} \times \mathrm{O}$ at the layer-half-space interface (point 3 in the finite difference grid, see Fig. 1) and point $T \quad x \quad H_{b} \quad{ }_{b} t_{d}=2$, where the amplitudes of the strain with the same sign meet after reflec- 

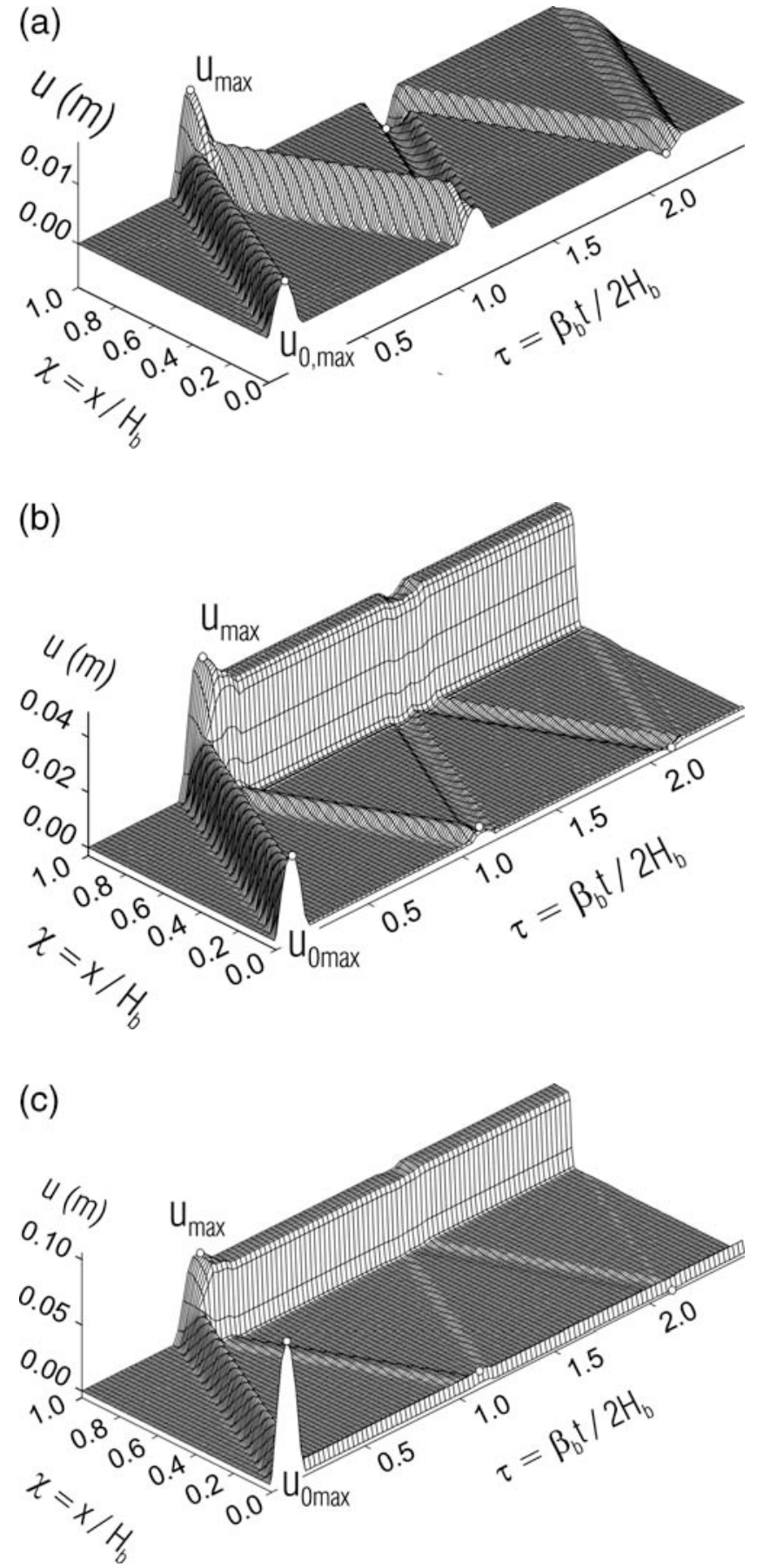

Figure 3. Comparison of displacements along the normalized length of the beam, $\quad \mathrm{x}=\mathrm{H}_{\mathrm{b}}$, versus normalized time ${ }_{b} t=2 \mathrm{H}_{b}$ for dimensionless frequency 3 for $(\mathrm{a})$ linear response when dimensionless pulse has amplitude 0:03 and nonlinear responses when dimensionless pulse has amplitude (b) 0:1 and (c) $\quad 0: 3$.

tion from the top of the layer. The location of this point depends upon the duration (wavelength) of the pulse. To find the location of this point and the time of occurrence of the local maximum strain, we use equation (15). The first term in equation (15) is one if the argument of the cosine function is equal to $t_{d} t \quad t_{0} \quad x={ }_{b} \quad t_{d}$, and the second term is one if the argument of the second cosine function is equal to zero $\left(\begin{array}{llll}t & t_{1} & x=b & 0\end{array}\right)$. We then solve the system of two equations for $x$ and $t$ bearing in mind $t_{0} \quad 0$ and $t_{1} \quad 2 H_{b}={ }_{b}$.

The position of point $\mathrm{T}$, where the rotation (strain) amplitude is two times larger than the rotation (strain) entering the layer, is at $x \quad H_{b} \quad b \cdot t_{d}=2$, and the time when this occurs is $t \quad H_{b}={ }_{b} \quad t_{d}=2$. From equation (15) during the first passage of the pulse $t<2 H_{b}=b$ and at point $B$, only the first term in the series exists. The strain at point $B$ reaches its absolute maximum at the very beginning during the entrance of the pulse into the layer, and its value is

$$
\text { j }{ }_{B \max }^{1} j \quad \pi \quad{ }_{y b} k_{t} \text { : }
$$

If the strain in equation (18) is greater than the yielding strain in the layer, a permanent strain at the interface will develop. The condition for occurrence of permanent strain at this point is $\mathrm{j}_{\mathrm{B} \text { max }}^{1} \mathrm{j}>\mathrm{yb}$ or in terms of the dimensionless parameters

$$
>\pi k_{t}{ }^{1} \quad \text { b b } \quad s s=2 \pi s s \quad C_{B}:
$$

At point $T$ (this point does not exist if $t_{d}>2 H_{b}={ }_{b}$, and it coincides with point $B$ if $t_{d} \quad 2 H_{b}={ }_{b}$ ) from equation (15), the maximum strain during the first passage occurs at $t$ $\mathrm{H}_{b}={ }_{b} \quad t_{d}=2$, and its amplitude is $2 \mathrm{~A} \cdot \mathrm{k}_{\mathrm{t}}$. The condition for occurrence of the permanent strain is

$$
>2 \pi \mathrm{k}_{\mathrm{t}} 1 \quad \text { b b } \quad \mathrm{s} s=4 \pi \mathrm{s} s \quad \mathrm{C}_{\mathrm{T}} \quad \mathrm{C}_{\mathrm{B}}=2 \text { : }
$$

For the shear-wave velocities in our example, $C_{B} \quad 0: 2228$ and $\mathrm{C}_{\mathrm{T}} \quad 0: 1114$.

When the reflected wave from the top of the layer reaches the soil-half-space interface $\left(t t_{1}\right)$, the wave begins the second passage. The linear solution for the strain in equation (15) at $B$ now involves three terms in the series if the duration of the pulse is longer than $2 \mathrm{H}_{b}={ }_{b}$ and two terms for shorter pulses. Recalling equations (15) and (17), the solution at time $t \quad 2 \mathrm{H}_{\mathrm{b}}={ }_{\mathrm{b}} \quad \mathrm{t}_{1}$ is

$$
0 ; t_{1} \quad A f k_{1} \quad \cos 2 \pi H_{b}=b_{b} t_{d} \quad \cos 0 \quad k_{2} \cdot \cos 0 g
$$

or

$$
0 ; t_{1} \quad \pi \quad y_{b} k_{t} 1 \quad \cos 2 \pi \quad k_{r}
$$

when $t_{d}>2 H_{b}=b \quad<1=2$, and

$$
0 ; t_{1} \quad A \quad k_{1} \cdot \cos 0 \quad k_{2} \cdot \cos 0 ;
$$

or

$$
0 ; t_{1} \quad \pi \quad{ }_{y b} k_{t} 1 \quad k_{r}
$$

when $t_{d}<2 H_{b}={ }_{b} \quad>1=2$.

Comparing equations (18) and (24) because $\mathrm{k}_{\mathrm{r}}<0$ for short pulses $(>0: 5)$, it is seen that the strain at point $B$ at 
the beginning of the second passage is always larger (1 $j k_{r} j$ ), and thus for our example, $10=7$ times larger than the strain $A \cdot k_{t}$ at the beginning of the first passage. However, it is still smaller than the strain at $T$ in the first passage $2 A \cdot k_{t}$. For short pulses $(>0: 5)$ it can be concluded that if there is no occurrence of permanent strain during the first passage at point $T$, the response of the layer will be linear for all time.

For long pulses $(<0: 5)$ comparing equations (18) and (22) and for $<2 \pi{ }^{1} \arccos j k_{r} j$, the strain at point $B$ at the beginning of the second passage is smaller than the strain at the beginning of the first passage, and for

$>2 \pi^{1} \arccos j k_{r} j$, the former strain is larger than the later one. For our example $\left(k_{r} \quad 3=7\right), \quad>0: 18$ always gives larger strain at the interface point at the beginning of the second passage than the strain at the beginning of the first passage.

The largest amplification of the rotation (strain) at B is for $\quad 0: 5$ when at the beginning of the second passage, the strain is (2 $\left.\quad j k_{r} j\right)$ times larger than the strain during the first passage. For our example the amplification is 17=7 ( $2: 43$ for $<0: 187)$, which gives larger strain than the strain at point $\mathrm{T}$ during the first passage $2 \mathrm{~A} \cdot \mathrm{k}_{\mathrm{t}}$. Therefore, for long pulses ( $\leq 0: 5)$ the first permanent strain can occur later in time. This means that in addition to conditions (19) and (20), there is one further condition for the occurrence of permanent strain, which is valid only in zone 1 , namely

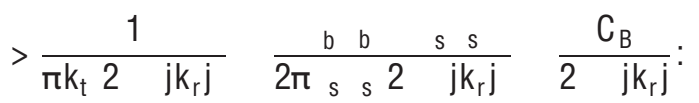

In further discussions, the region $; x$ will be divided into three zones-zone $1: Z_{1} \quad ; x j<0: 5 ; x$, zone $2: Z_{2} \quad ; x j 0: 5 \leq ; x \quad 0$, and zone $3: Z_{3}$

$; x j 0: 5 \leq ; x>0$. In the numerical simulations in this article, the boundary between zones 2 and 3 is taken close to the layer-half-space contact, and for convenience we have selected it to be at $\mathrm{x} 1$.

In the following, we consider the normalized maximum strain in the layer, $\max$. This strain is the absolute maximum of the strain occurring in the layer at any time of its response. To represent it in dimensionless terms, we consider the quantity $v_{\text {lin }} \quad v_{\text {entr }}^{\text {lin }}$, which is the maximum velocity entering the layer (supposing that it is linear) for the considered frequency of the pulse. The $v_{\text {lin }}$ is linear in , which follows from

$$
v_{\text {lin }} \quad \pi A \cdot k_{t}=t_{d} \quad \pi \quad y b \quad b k_{t}:
$$

Then, instead of describing the absolute maximum of the strain, we will consider the normalized maximum strain,

$$
\max _{\text {norm }} \quad \max \quad b=V_{\text {lin }} \quad \max ={ }_{\text {lin }} \text { : }
$$

This quantity will show the degree of nonlinearity in the layer response and the effects of the interference on the amplification of the linear entry strain. This strain is always larger than one. We will also describe the normalized strain at the end of the analysis in terms of the ratio

$$
\text { end }_{\text {norm }} \quad \text { end } b=V_{\text {lin }} \quad \text { end }={ }_{\text {lin }} \text { : }
$$

This quantity will show the amplitude of the permanent strain (rotation) after all of the wave energy exits the layer, relative to the linear entry strain. This strain can be larger or smaller than one, and for linear waves (when neither condition 20 nor condition 25 are satisfied), it is zero. We will also consider the maximum strain normalized by the yielding strain

$$
\underset{\text { norm }}{y} \quad \max ={ }_{y b} \text { : }
$$

In analogy with the response studies of engineering structures, if this quantity at some point of the soil layer is larger than the maximum allowed ductility fail $=y b$, where fail is the largest strain that can occur in the system, the layer may collapse. For linear waves this normalized strain is smaller than one.

For $0: 01$, the condition (25) is not satisfied in the zone $Z_{1}(\leq 0: 5)$ because in our example > $\mathrm{C}_{\mathrm{B}}=17=7 \quad 0: 2228 \cdot 7=17 \cdot 0: 01 \quad 9: 174>0: 5$, and so there is no permanent strain in this region. The condition (20) gives $>\mathrm{C}_{\mathrm{B}}=2 \quad 0: 2228=2 \cdot 0: 01$ $11: 14>5$, which means that in the range $0: 06 \leq \leq 5$, the response of the layer for 0:01 is always linear. The absolute maximum occurs in zone $Z_{1}$ for close to 0.5 at the bottom of the layer. Its value is 2.43 . For $>0: 5$ this normalized strain is constant and equal to two. In zone $Z_{2}$ the largest normalized strain occurs at the beginning of the second wave passage, and its theoretical value in our example is $1 j k_{r} j \quad 10=7 \quad 1: 43$. The finite difference calculations also give 1.43 .

To illustrate the dependence of the strain (rotations) on , we show Figure 4, where the propagation of the displacement along the dimensionless length of the layer $\quad \mathrm{x}=\mathrm{H}_{\mathrm{b}}$ in dimensionless time $\quad b t=2 H_{b}$ is shown for the first two and a half passages of the wave on the path bottom-topbottom of the layer. The dimensionless amplitude is $0: 3$, and the dimensionless frequency is 3 . At the top of this figure the motion is shown for elastoplastic material,

$0: 0$, and at the bottom for material with 0:3. Comparing the top and bottom plots, it is seen that increasing reduces the strain. Also, large strains for $0: 0$ are localized, while for $\quad 0: 3$ the nonlinear zone has a transition zone, which results from spreading of the zone with strain localization when is greater than zero. This is because for the modulus $1 \quad 0>0$ (Fig. 2 ), the permanent strain can propagate with velocity b1 $\mathrm{N}_{-}$. $\mathrm{b}$. For elastoplastic material 0 the permanent strain does not propagate but is accumulated in narrow zones of the layer, and the value of the strain at the point changes only when the pulse occupies the point. When $>0$ with propagation of the permanent strain, there is less accumulation of large strains at a point 


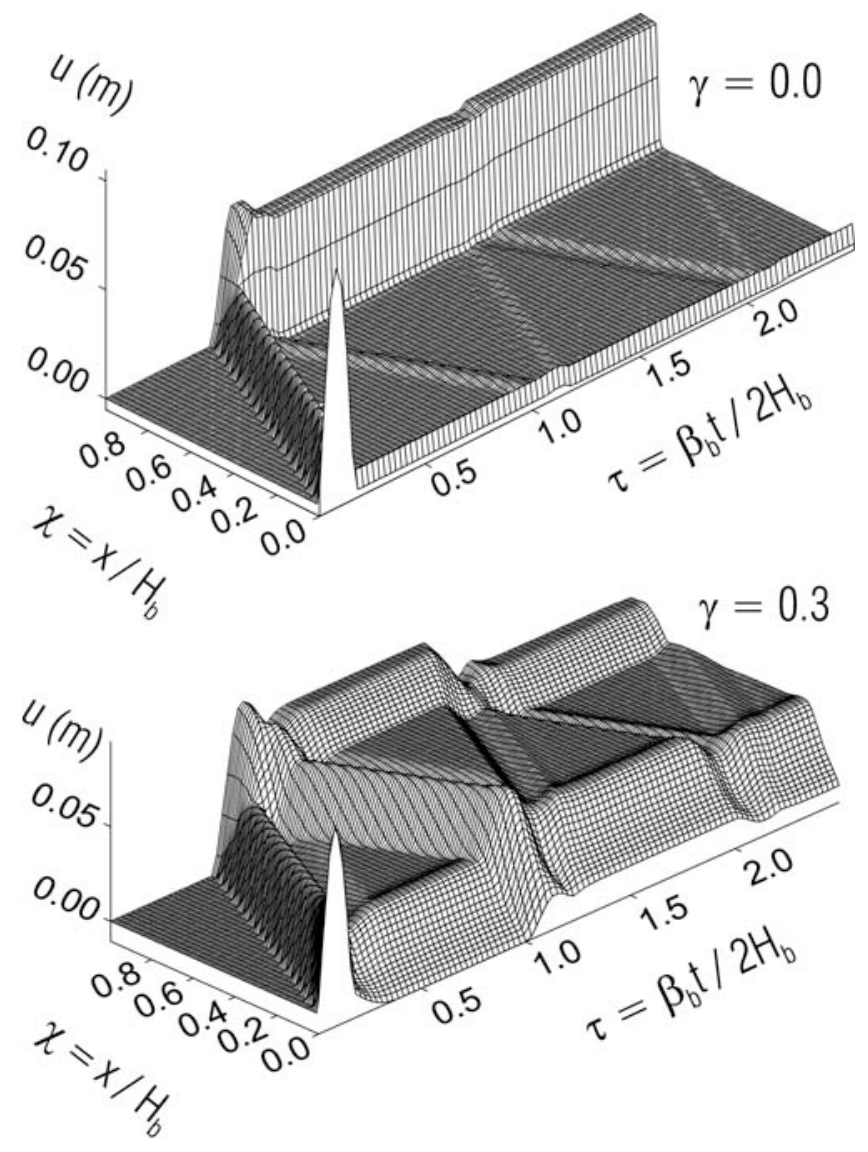

Figure 4. Plot of the displacements along the normalized length of the beam, , versus normalized time, , for dimensionless amplitude, $\quad 0: 3, \quad 3$, and for $\quad 0$ and $0: 3$.

with each passage of the wave through that point. Thus, as increases, the strain ordinates decrease, and the zones of the permanent strain become wider.

In Figure 4 the maximum strain occurs at the beginning of the wave entering the layer. For elastoplastic material, this strain is high. After reflecting from the top, almost the whole energy of the input wave is captured and spent for developing permanent strain in narrow zones, and only elastic strain continues to propagate along the layer. For the material with $0: 3$ with the development of permanent strain at the top, this strain does not remain at the point but propagates with velocity b1, which causes a substantial amount of energy to continue to spread out, up, and down the layer. For longer pulses this is not so obvious (Gi ev and Trifunac, 2007b). The permanent strain occurs in the beginning of the second passage of the wave along the layer thickness. It can be shown that for the elastoplastic material the permanent strain at the bottom is large while for the material with $\quad 0: 3$, this permanent strain may not even be noticeable, and it may appear as if the layer is in a linear state.

Figure 5 illustrates the space-frequency $(x)$ dependence of normalized peak strain $\max b=V_{\text {entr }}^{\text {in }}$ for four normalized excitation amplitudes $0: 05,0.1,0.2$, and 0.3 . The normalized strain in the soil layer is plotted at the instant when the absolute maximum of the normalized strain occurs for a given frequency . This is done for all frequencies considered in this work. Figure 6 illustrates the dependence of $\max b=v_{\text {entr }}^{\text {lin }}$ on $\quad 0: 0,0.1,0.2$, and 0.3 for the normalized amplitude of excitation $\quad 0: 1$. Figures 5 and 6 also show the peak amplitudes in zones 1,2 , and 3 .

The normalized strains versus dimensionless amplitude are illustrated in the subsequent figures for the four values of $\quad 0: 0,0.1,0.2$, and 0.3 in the three zones $Z_{1}, Z_{2}$, and $Z_{3}$, respectively, using a semilogarithmic scale. From condition (25), the first nonlinear strain in zone 1 occurs for

$$
{ }_{1}>0: 09174={ }_{\max } \quad 0: 09174=0: 5 \quad 0: 1835:
$$

As can be seen in zone 1 (Fig. 7a), while the strain is linear $(\leq 1)$, there is no dependence upon, and all of the curves coincide. The normalized maximum strain $\underset{\text { norm }}{\max }$ (see equation 27) is constant, and for our example its value is $17=7 \quad 2: 43$ corresponding to the summation of the three strains at the beginning of the second passage. Because the response is linear, in this interval max shows only the effect of the interference on amplification of the linear entry strain. The normalized end strain end in this interval is zero, showing that the strains are reversible and that the resistance capacity of the layer for some future excitation is not diminished. The normalized strain ${ }_{n}^{y} \quad \max =y b$ approaches zero as approaches zero. With increasing beyond 1 , the response at the bottom of the layer becomes nonlinear at the beginning of the second passage, the curves for different values of separate, and the normalized strains increase with decreasing being the largest for elastoplastic material, $\quad 0$ : The normalized strain $\max _{\text {norm }}$ reaches its maximum values for largest shown in the plots for elastoplastic material. In this case the effect of the nonlinearity of the layer response dominates over the effect of constructive interference of the three strains at the bottom. After the wave exits the layer completely, the remaining normalized permanent strain at the end end has almost the same amplitudes for the elastoplastic material, and the normalized strain ${ }_{\text {norm }}^{y}$ is also larger, indicating that the layer will fail at large values of .

In Figure $7 \mathrm{~b}$ the normalized strains versus in zone 2 are shown again using the semilogarithmic scale. It can be seen that for small amplitudes $\leq 0: 05$, all of the curves converge to zero. As increases, all normalized strains increase. For larger than 0.1 , the dependence of all of the normalized strains on for elastoplastic material 0 in this scale resemble a logarithmic function, which means that the normalized strains for elastoplastic material are linear functions of . For materials with $\neq 0$, the normalized strains $\begin{gathered}\text { end } \\ \text { norm }\end{gathered}$ normalized strains $\max _{\text {norm }}$ and $y$ norm are approximately linear with the slope $\partial \max _{\text {norm }}=\partial$ smaller than the slope $\partial \underset{\text { norm }}{y}=\partial$.

In Figure $7 c$ the normalized strains versus in zone 3 are shown. For any the normalized strains $\max _{\text {norm }}$ and $\underset{n}{y}$ approach 2 and 0 , respectively, as approaches 0 . The nor- 


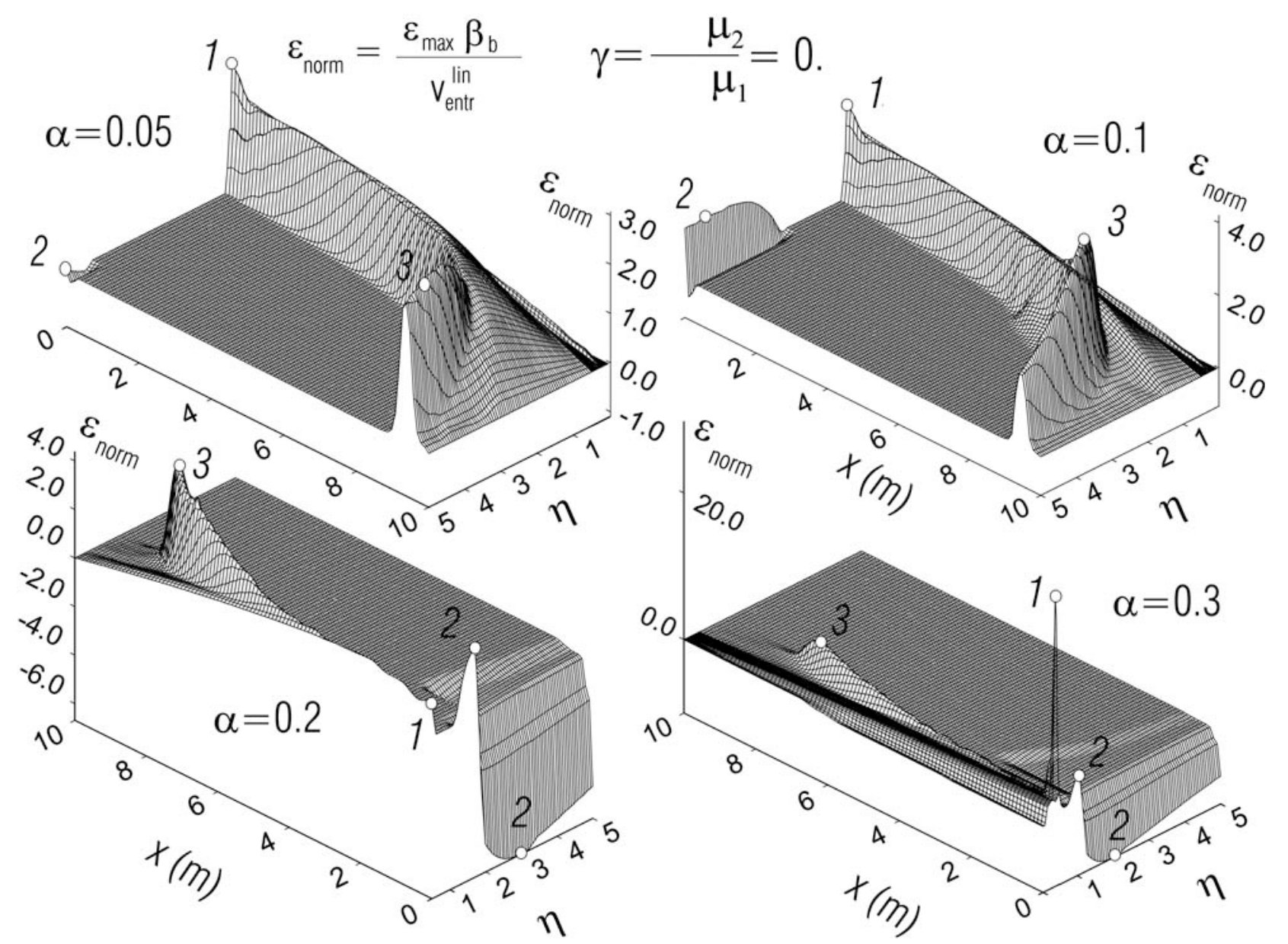

Figure 5. Normalized strains along the beam when its maximum occurs versus dimensionless frequency, , for 0 and for four dimensionless amplitudes.

malized strain $\underset{\text { norm }}{\text { end }}$ depends only on and is independent of . The lowest amplitude when the permanent strain occurs can be found from condition (20) at the highest considered as $\min >0: 1114=5 \quad 0: 02228$. For $\leq \min$, end 0 norm

Figure 8 shows the zones of the layer in which the maximum strains (rotations) occur versus the dimensionless amplitude of the strong-motion pulse in the range from 0.0 to 0.6 , for $\quad 0: 0$ and 0.3 . For all normalized strains, $\max b=V_{\text {lin }}$, end $b=V_{\text {lin }}$, and ${ }_{\max }={ }_{y b}$, and for increasing from 0 , essentially all peaks first occur in zone 3 of the layer. The only exception to this for $\max b=V_{\text {lin }}$ is for small values of for which the response is linear and for which the peak of this strain occurs in zone 1 . For $\max _{y b}$ and for $0: 0$ beyond $\quad 0: 15$, the peak rotations begin to occur in zone 2 with negative signs for all $<0: 6$ considered in this article. For $\quad 0: 3$ beyond $\quad 0: 1$, the peak rotations $\max _{\mathrm{yb}}$ begin to occur in zone 2 and are negative up to 0:25; they change sign to positive for beyond 0.25 for all $<0: 6$ considered in this article.

For the largest normalized pulse amplitudes we considered in this work, $\quad 0: 6$, and assuming motions close to the moving fault such that $\mathrm{v}_{\text {lin }} 100 \mathrm{~cm}=\mathrm{sec}$, for example, the results in Figure 8 imply permanent shearing of the parts of the layer, which would lead to permanent tilting of the deep embedded foundations in the range from $0: 6^{\circ}$ (when

\section{$0: 3$ ) to $57^{\circ}$ (when 0:0). The latter result for}

$0: 0$ exceeds the range of the response amplitudes ( $\tan$ ), which can be calculated reliably with our model, but it shows nevertheless that the strains can become very large. The adverse consequences of such large shearing deformations on deep foundations and foundations on piles are obvious.

\section{Discussion and Conclusions}

In this article we described the consequences of earthquake energy flow into a soil layer with the objective of providing some understanding of the resulting response for excitation by powerful transient pulses. Starting with a linear strong-motion pulse in the half-space and ending with nonlinear waves propagating through a soil layer, we identified the peak transient and permanent rotations (strains) occurring during various stages of excitation.

For incident short pulses $(>0: 5)$, which lead to linear response of the layer, the amplification of a pulse with normalized pulse amplitude $\quad\left[\quad A=H_{b}\right.$ yb and represents a 


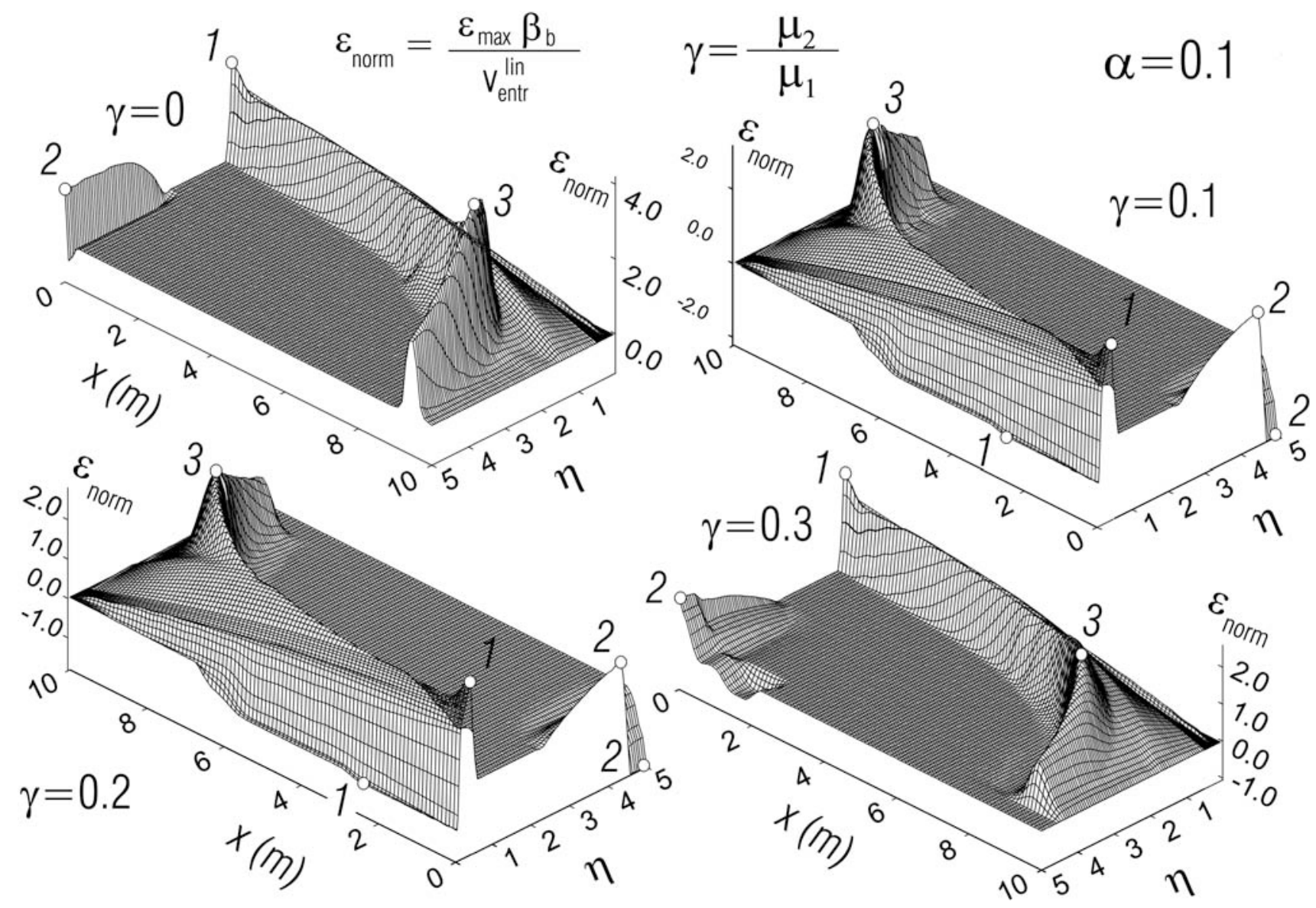

Figure 6. Normalized strains along the beam when its maximum occurs versus dimensionless frequency, , and for dimensionless amplitude,

ratio of the average drift in the layer $A=H_{b}$ and of the yielding strain in the soil material $\mathrm{yb}$ ] is equal to two. It results from interference of the upward-propagating wave with the wave reflected from the free layer surface and propagating down. For long pulses ( $\leq 0: 5)$, the amplification depends upon the impedance ratio between the half-space and the layer material, and depending upon the duration of the pulse, it can occur during first, second, or higher-order passes of the wave up and down the layer. In the example presented in this article, it occurs at the beginning of the second pass and is equal to 2.43 .

For large values of , which lead to nonlinear wave motion in the layer, amplification of peak rotations (strains) is a strong function of the second slope in the bilinear representation of the stress-strain relationship of the layer. For

0:0 the amplification of all peak rotations and strains grows rapidly with while for 0:3 and larger, it is only slightly above the amplification for linear wave motion.

With increasing and the first appearance of the nonlinear response, the maxima of all normalized strains $\max b=V_{\text {lin }}$, end $b=V_{\text {lin }}$, and $\max _{y b}$ first occur in zone 3 of the layer ( $>0: 5$ and $0<x \leq H_{b}$; Fig. 8). This corresponds to interference of up- and downward-propagating short waves after reflection from the layer surface, and it occurs for in the vicinity of 0.2. Beyond $0: 2$, the peaks of $\max _{\mathrm{yb}}$ occur just above the interface of the layer and the half-space (in zone 2: $>0: 5$, and $x \quad 0$ ). The peaks of $\max b=V_{\text {lin }}$ and end $b=V_{\text {lin }}$ occur in zone $3(>0: 5$, and $\left.0<x \leq H_{b}\right)$ for $\quad 0: 3$ and in zone $1(\leq 0: 5$, and $x)$ for $0: 0$.

The description of the peak rotations and peak strains in nonlinear response is for the example of soil layer in this article only. For layers with constant shear-wave velocities, densities, and yb different than those considered in our example, the scales of the coordinate axis in Figure 7a,b,c and Figure 8 will shrink or extend, but the overall nature of the results will remain similar. For the layers with variable shearwave velocities and densities, the general appearance of the peaks of $\max b=V_{\text {lin }}$, end $b=V_{\text {lin }}$, and ${ }_{\max }={ }_{y b}$ will remain similar, but they may include additional complexities, which will result from reflection and refraction from the jumps caused by changes in the shear-wave velocity. Some aspects of those complexities can be seen in the related analysis of the nonlinear waves in a seven-story hotel building in Van Nuys, California, which was damaged by the 1994 Northridge earthquake (Gi ev and Trifunac, 2007a). 

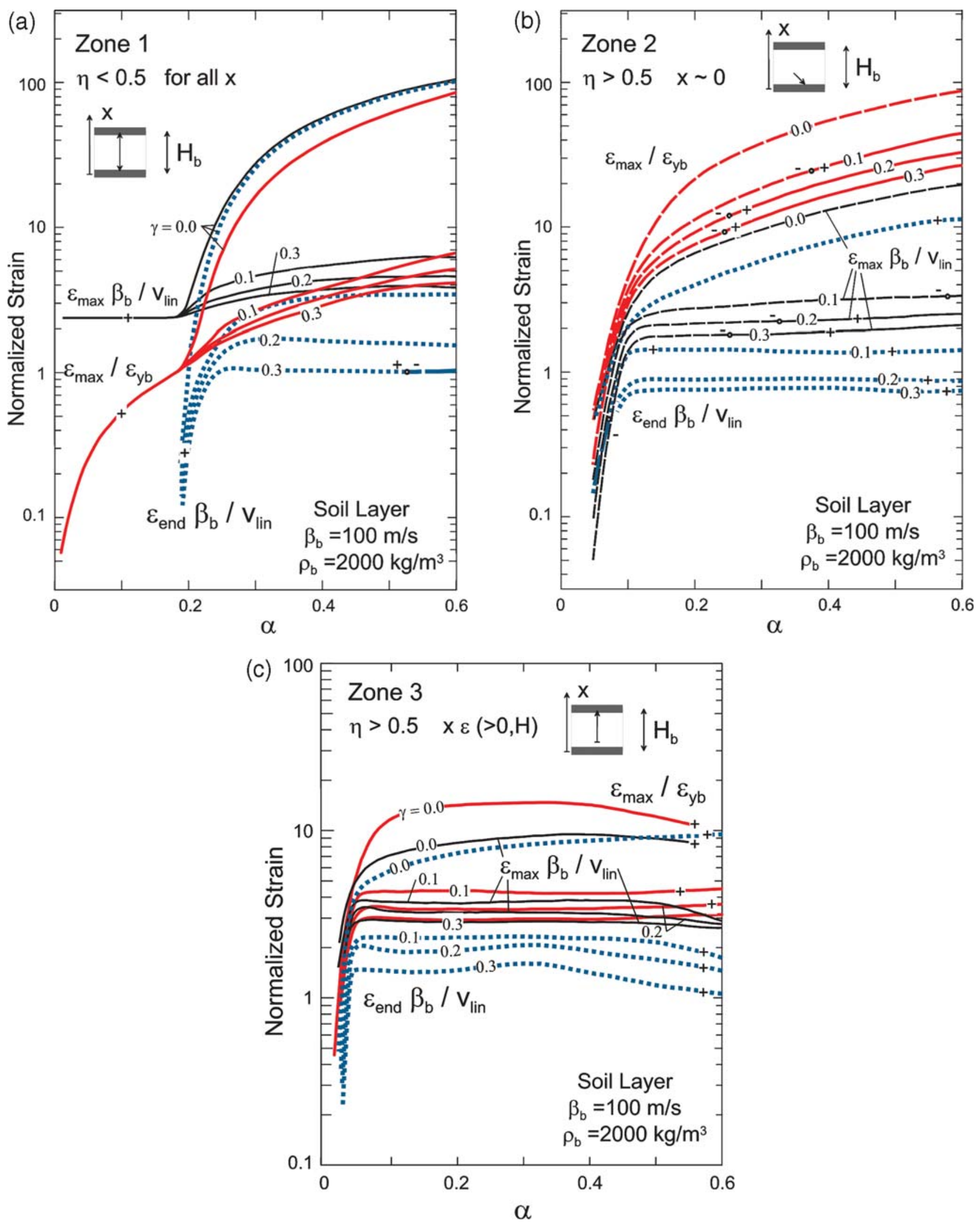

Figure 7. Normalized strains versus the dimensionless amplitude, , for four different values of $\quad 0: 0,0.1,0.2$, and 0.3, in (a) zone 1 , (b) zone 2, and (c) zone 3 . 


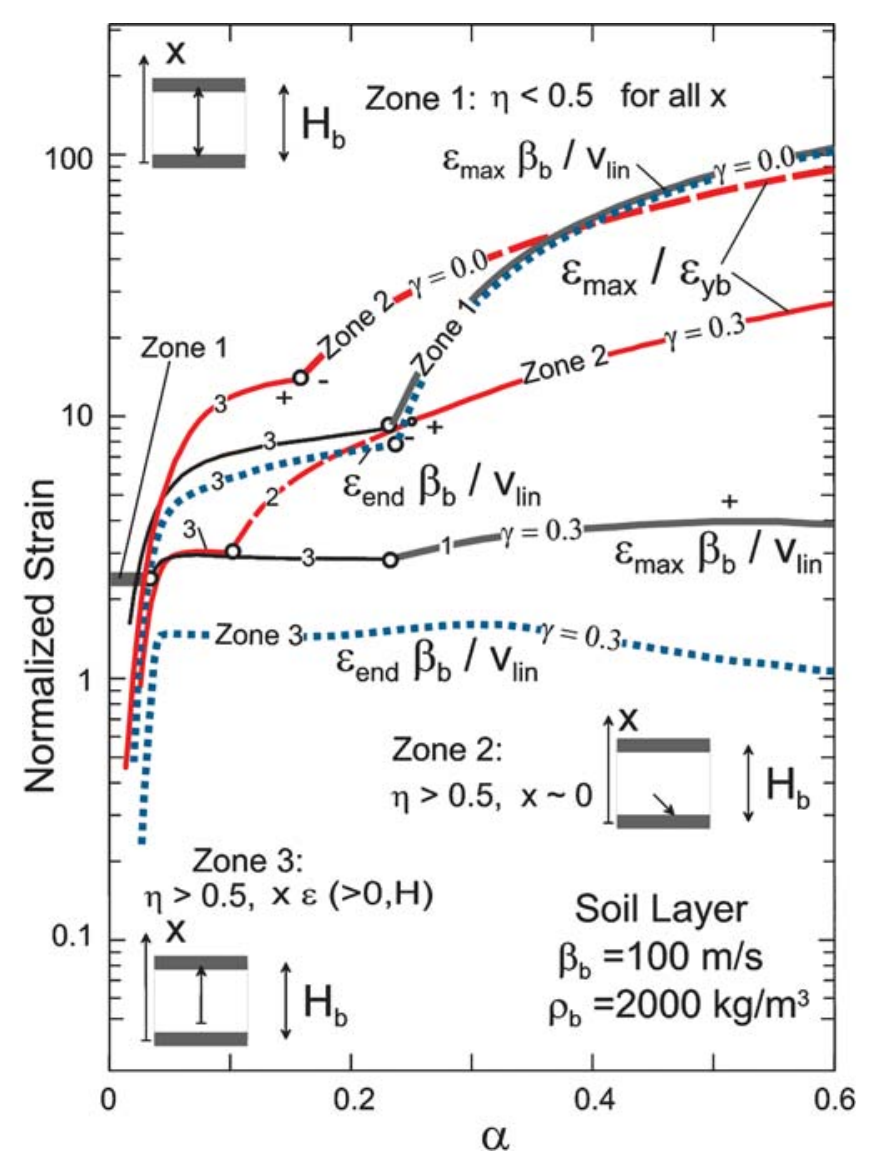

Figure 8. Dependence of the largest normalized strains versus the dimensionless pulse amplitude, , showing the zone where the largest peak occurs for $0: 0$ and 0.3 .

The strain localization, which can occur almost any place in the layer depending upon the and of the pulses (as the examples discussed previously show), implies that liquefaction and lateral spreading, for example, can be initiated at any depth of the layer. Thus, the final outcome will always depend upon the nature of the excitation and upon how many energetic peaks, and of what sizes, are present in the train of strong ground motion (Todorovska et al., 2008).

A review of Figure 7a,b,c might suggest that the normalized peak rotations (strains), $\max b=V_{\text {lin }}$, end $b=V_{\text {lin }}$, and $\max _{\mathrm{yb}}$ converge to an asymptote or to a monotonically increasing trend when $\rightarrow \infty$. However, this is not the case. First, positive and negative peak strains dominate the maxima in a manner that is not simple. In zones 1 and 3 , most maxima are positive, but in zone 2 positive and negative peaks appear and disappear in a manner that is not simple and recognizable. Second, with increasing beyond 0:2 in our example, what at first would seem to be a monotonic trend begins to fluctuate, and in some instances the peak disappears. This can be seen for the curves in Figure 7c, for example, where some curves are interrupted near 0:5 (for $\max b=V_{\text {lin }}$ and for $\max _{y b}$, both for 0:0), some suddenly begin to decrease beyond $0: 4 \quad\left(\max _{b}=V_{\text {lin }}\right.$ for $\quad 0: 1,0.2$, and 0.3 ), and some just oscillate up and down departing from a monotonic trend ( end $b=\mathrm{V}_{\text {lin }}$ for

$0: 1,0.2$, and 0.3 ). Our numerical algorithm has been formulated to work with small strains when tan, and so we cannot obtain reliable results for large nonlinear deformations. Furthermore, the differential equations we chose for describing 1D shear waves are also linear and do not include higher-order terms associated with geometric nonlinearities, gravity effects, and dynamic instabilities. Yet the nature of the problem we study is characterized by large nonlinearities, and therefore, it is expected to display the characteristics of chaotic response.

The completeness of the linear differential equations has led to their dominance in the mathematical training of engineers and seismologists during most of the twentieth century, which means that the recognition that chaotic motions are inherent in most nonlinear physical phenomena has had little, if any, effect on the research in earthquake engineering thus far. In the past, the analysis of site response has been kept mainly within the realm of linear or equivalent linear mechanics (Safak, 2001; Hartzell et al., 2004). However, if an engineer chooses parameters that produce chaotic output, he loses reliable predictability. The chaotic behavior of nonlinear systems does not exclude predictability of the response but rather introduces upper bounds (prediction horizons) (Lighthill, 1994) and renders the predictions probabilistic. The important question is then over what timescale the forecasts are reliable given the current state and knowledge of the system. Another key ingredient for prediction is an adequate physical model. At present, because of the multitude of interacting phenomena and the absence of physically complete equations of motion, there exists no adequate general model of the complete earthquake response process; and therefore, the practical outcome of most work in earthquake engineering remains empirically based.

The aforementioned information implies that there is a conflict in the classical engineering description of the world. One aspect of this conflict is the assumption that nature is moving forward according to deterministic laws. Another is that an engineer attempting to model portions of the world from finite data projects unverifiable structure onto the local environment. The conflict is that these two views do not match, leading to a question, What are models good for? There are many systems in nature that are observed to be chaotic and for which no adequate physical model exists. Whether a model is adequate or not depends, of course, on the questions asked (Crutchfield, 1992). Unfortunately, the art of dynamical modeling is often neglected in discussions of nonlinear and chaotic systems, in spite of its crucial importance (Beltrami, 1987).

Stochastic processes have been introduced to describe irregular phenomena in deterministic systems that are too complicated or have too many variables to be fully described in detail. For example, stochastic processes have been used to model site response to earthquake shaking, which is deterministic and thus, in principle, could be completely de- 
scribed. In practice, the stochastic modeling has also been used as an approximate description of a deterministic system, which has unknown initial conditions and may be highly sensitive to the initial conditions. In trying to model real systems, as a result of the modeling process, we sometimes obtain a model that shows very regular behavior while the real system has very irregular behavior. In that case random noise is added to the model, but this represents no more than our lack of knowledge of the system structure or the inadequacy of the identification procedure (Kapitaniak, 1991; Biot, 2007).

It has been argued that advances in science occur not so much when new theories are advanced as when the simple models with which scientists conceptualize a theory are changed (Kuhn, 1962). In the analysis of site conditions, such a conceptual model that embodies the major features of a whole class of problems is the single layer of soil over a semi-infinite half-space. Lessons emerging from studies of this simple model may serve as conceptual starting points for generalizations and also as a guide to further studies of more complex models in earthquake engineering and strongmotion seismology.

\section{Data and Resources}

No data were used in this article. Parts of some of the plots used in this article came from published sources listed in the references.

\section{References}

Beltrami, E. (1987). Mathematics for Dynamic Modeling, J. Wiley and Sons, New York.

Biot, M. A. (2007). On the concept of response spectrum in engineering seismology, in Early History of the Response Spectrum Method, M. D. Trifunac (Editor), Dept. of Civil Engineering, Report CE 07-01, University of Southern California, Los Angeles, California.

Bonilla, L. F. (2000). Computation of linear and nonlinear site response for near field ground motion, Ph.D. Thesis, University of California, Santa Barbara, California.

Crutchfield, J. P. (1992). Knowledge and meaning, in Modeling Complex Phenomena, L. Lam and V. Naroditsky (Editors), Springer-Verlag, New York, 66-101.

Earthquake Engineering Research Institute (EERI) (1995). Northridge Earthquake of January 17, 1994, Reconnaissance Report, Earthquake Spectra, Supplement C to Vol. 11, Vol. 1.

Finn, W. D. L. (1982). Dynamic response of saturated sands, in Soil Mechanics: Transient and Cyclic Loads, G. N. Pande and O. C. Zienkiewicz (Editors), J. Wiley and Sons, New York, 105-131.

Fujino, Y., and M. Hakuno (1978). Characteristics of elasto-plastic ground motion during an earthquake, Tokyo University, Bull. Earthq. Res. Inst. 53, 359-378.

Gi ev, V., and M. D. Trifunac (2007a). Energy and power of nonlinear waves in a seven story reinforced concrete building, Indian Soc. Earthq. Technol. J. 44, no. 1, 305-323.

Gi ev, V., and M. D. Trifunac (2007b). Permanent deformations and strains in a shear building excited by a strong motion pulse, Soil Dyn. Earthq. Eng. 27, no. 8, 774-792.

Hardin, B. O., and V. P. Drnevich (1972). Shear modulus and damping in soils: Design equations and curves, Proc. Am. Soc. Civil Eng. 98, 667-692.
Hartzell, S., L. F. Bonilla, and R. Williams (2004). Prediction of nonlinear soil effects, Bull. Seismol. Soc. Am. 94, no. 5, 1609-1629.

Iai, S., Y. Matsunaga, and T. Kameoka (1990). Strain space plasticity model for cyclic mobility, Rep. Port Harbor Res. Institute, Vol. 29, $27-56$.

Joyner, W. B., and A. T. F. Chen (1975). Calculation of nonlinear ground response in earthquakes, Bull. Seismol. Soc. Am. 65, no. 5, 1315-1366.

Kanai, K. (1965). Some problems of seismic vibration of structures, Proc. of the Third World Conf. on Earthquake Engineering, New Zealand, II, 260-275.

Kanai, K. (1983). Engineering Seismology, University of Tokyo Press, Tokyo, Japan.

Kapitaniak, T. (1991). Chaotic Oscillations in Mechanical Systems, Manchester University Press, Manchester, United Kingdom.

Kramer, S. L. (1996). Geotechnical Earthquake Engineering, Prentice Hall, New York.

Kuhn, T. (1962). The Structure of Scientific Revolutions, The University of Chicago Press, Chicago, Illinois.

Lax, P. D., and B. Wendroff (1964). Difference schemes for hyperbolic equations with high order of accuracy, Commentary XVII, 381-398.

Li, X., and Z. Liao (1993). Dynamic skeleton curve of soil stress-strain relation under irregular cyclic loading, Earthq. Res. China 7, 469-477.

Lighthill, J. (1994). Chaos: A historical perspective, in Nonlinear Dynamics and Predictability of Geophysical Phenomena, W. I. Newman, A. Gabrielov, and D. Turcotte (Editors), Geophysical Monograph 83, Vol. 18, IUGG, 1-5.

Massing, G. (1926). Eigenspannungen und Verfestigung beim Messing, in Proc. of Second International Congress of Applied Mechanics, Zurich, Switzerland, 332-335.

Milne, J. (1898). Seismology, Kegan, Trench, and Truber, London.

Pyke, R. M. (1979). Nonlinear models for irregular cyclic loading, J. Geotech. Eng. Div., ASCE, 105, no. GT6, 715-726.

Safak, E. (2001). Local site effects and dynamic soil behavior, Soil Dyn. Earthq. Eng. 21, no. 5, 453-458.

Seed, H. B., and I. M. Idriss (1970). Soil moduli and damping factors for dynamic response analysis, Earthquake Engineering Research Center, University of California, Berkeley,Report EERC 70-10.

Sezawa, K., and K. Kanai (1935). Decay in the seismic vibration of a simple or tall structure by dissipation of their energy into the ground, Tokyo University, Bull. Earthq. Res. Inst. XIII, no. 3, 681-697.

Sezawa, K., and K. Kanai (1936). Improved theory of energy dissipation in seismic vibrations on a structure, Tokyo University, Bull. Earthq. Res. Inst. XIV, no. 2, 164-168.

Takeo, M. (2006). Ground rotational motions recorded in near-source region of earthquakes, Earthquake Source Asymmetry, Structural Media and Rotation Effects, R. Teisseyre, M. Takeo, and E. Majewski (Editors). Springer, Heidelberg, Germany, 157-167.

Takeo, M., and H. I. Ito (1997). What can be learned from rotational motions excited by earthquakes?, Geophys. J. 129, 319-329.

Terzaghi, K. (1925). Erdbaumechanik auf bodenphysikalisher Grundlage, Deuticke Verlag, Leipzig und Wien.

Todorovska, M. I., H. Meidani, and M. D. Trifunac (2008). Wavelet approximation of earthquake strong ground motion-Goodness of fit for a database in predicting nonlinear structural response, Soil Dyn. Earthq. Eng., doi 10.1016/j.soildyn.2008.05.011.

Trifunac, M. D. (2008). 75th anniversary of strong motion observationA historical review, Soil Dyn. Earthq. Eng., doi 10.1016/j.soildyn .2008.05.011.

Trifunac, M. D. (2009). The role of strong motion rotations in the response of structures near earthquake faults, Soil Dyn. Earthq. Eng. 29, no. 2, 382-393

Trifunac, M. D., and S. S. Ivanovi (2003a). Recurrence of site specific response in former Yugoslavia-Part I: Montenegro, Soil Dyn. Earthq. Eng. 23, no. 8, 637-661.

Trifunac, M. D., and S. S. Ivanovi (2003b). Reoccurrence of site specific response in former Yugoslavia-Part II: Friuli, Banja Luka, and Kopaonik, Soil Dyn. Earthq. Eng. 23, no. 8, 663-681. 
Trifunac, M. D., and M. I. Todorovska (1996). Nonlinear soil response1994 Northridge, California, earthquake, J. Geotech. Eng., ASCE 122, no. 9, 725-735.

Trifunac, M. D., and M. I. Todorovska (1998). Nonlinear soil response as a natural passive isolation mechanism-the 1994 Northridge, California earthquake, with M.I. Todorovska, Soil Dyn. Earthq. Eng. 17, no. 1, 41-51.

Trifunac, M. D., and M. I. Todorovska (2000a). Can aftershock studies predict site amplification? Northridge, California, earthquake of 17 January 1996, Soil Dyn. Earthq. Eng. 19, no. 4, 233-251.

Trifunac, M. D., and M. I. Todorovska (2000b). Long period microtremors, microseisms and earthquake damage: Northridge, California, earthquake of 17 January 1994, Soil Dyn. Earthq. Eng. 19, no. 4, 253-267.

Trifunac, M. D., and M. I. Todorovska (2004). 1971 San Fernando and 1994 Northridge, California, earthquakes: Did the zones with severely damaged buildings reoccur?, Soil Dyn. Earthq. Eng. 24, no. 3, 225-239.

Trifunac, M. D., T. Y. Hao, and M. I. Todorovska (1999). On reoccurrence of site specific response, Soil Dyn. Earthq. Eng. 18, no. 8, 569-592.

Trifunac, M. D., S. S. Ivanovi , and M. I. Todorovska (2001a). Apparent periods of a building I: Fourier analysis, J. Struct. Eng., ASCE 127, no. $5,517-526$.
Trifunac, M. D., S. S. Ivanovi , and M. I. Todorovska (2001b). Apparent periods of a building II: Time-frequency analysis, J. Struct. Eng., ASCE 127, no. 5, 527-537.

Trifunac, M. D., M. I. Todorovska, and S. S. Ivanovi (1996). Peak velocities, and peak surface strains during Northridge, California, earthquake of 17 January 1994, Soil Dyn. Earthq. Eng., 15, no. 5, 301-310.

Vu eti , M. (1990). Normalized behavior of clay under irregular cyclic loading, Can. Geotech. J. 27, 29-46.

Rudarsko-Geološki Fakultet

Goce Del ev 89, 2000 Štip

Republic of Macedonia

(V.G.)

Department of Civil Engineering

University of Southern California

Los Angeles, California, 90089

(M.D.T.)

Manuscript received 15 May 2008 